\title{
Evaluación de la eficacia y seguridad de tigeciclina en el tratamiento de infecciones del tracto respiratorio. Revisión sistemática de la literatura científica
}

\author{
Patricia Moya Cordero, Jesús Ruiz-Aragón, Juan Máximo Molina Linde, \\ Sergio Márquez-Peláez y Virginia Motilva Sánchez
}

\section{Evaluation of the efficacy and safety of tigecycline for treatment of respiratory tract infections. Systematic review of literature}

Background: Tigecycline is indicated for the treatment of complicated skin infections, soft tissue and intraabdominal infections. Its use could be extended to community-acquired pneumonia (CAP) and hospital pneumonia $(\mathrm{HN})$. The objective was to evaluate the efficacy and safety of tigecycline in the treatment of respiratory infections. Methods: systematic review (2012). Databases used were MEDLINE, EMBASE, Cochrane Library, CRD and WOK. We identified clinical trials of adults with respiratory infection, treated with tigecycline. The quality of the studies was assessed using CASPe checklist. Results: We selected four clinical trials of high-moderate quality. Three studies with patients with CAP and a trial with $\mathrm{HN}$ patients. In patients with CAP, efficacy of tigecycline (88.6 to $90.6 \%)$ was higher than levofloxacin $(85.3$ to $87.2 \%)$. The non inferiority testing was statistically significant ( $p<$ 0.001 ). In the study of patients with HN tigecycline showed an efficiency of $67.9 \%$ versus $78.2 \%$ for imipenem/ cilastatin. Main adverse effects were gastrointestinal. Conclusions: The efficacy of tigecycline is non inferior than levofloxacin in patients with CAP, but less than imipenem in patients with HN. Tigecycline demonstrates noninferiority versus others tested antibiotics, and it shows a good safety profile.

Key words: Tigecycline, nosocomial pneumonia, community-acquired pneumonia, systematic review.

Palabras clave: Tigeciclina, neumonía nosocomial, neumonía adquirida en la comunidad, revisión sistemática.

Comentario Editorial en pág 598

\section{Introducción}

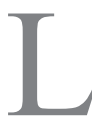

as infecciones del tracto respiratorio inferior son muy frecuentes, tanto las adquiridas en el ambiente comunitario como en el medio hospitalario ${ }^{1}$. La neumonía adquirida en la comunidad (NAC) constituye una causa muy importante de morbilidad y mortalidad. En Chile la neumonía constituye la principal causa de muerte por enfermedades infecciosas ${ }^{2}$ y la tercera causa específica de muerte en población adulta ${ }^{3}$. La incidencia y letalidad de NAC se elevan en lactantes bajo un año de edad y en mayores de 65 años. Así, la tasa de mortalidad por neumonía en la población anciana alcanza a 6,6 muertes por cada 1.000 habitantes $^{3}$. En España, la incidencia de la NAC en adultos se sitúa entre 2-10 casos por 1.000 habitantes $^{4}$, cifra que se eleva a $25-35$ casos en mayores de 70 años $^{5}$. El tratamiento antimicrobiano de la NAC se ha visto amenazado en los últimos años por la aparición y diseminación de cepas de Streptococcus pneumoniae con sensibilidad disminuida a penicilina y a otros antimicrobianos ${ }^{6}$. En la actualidad, la utilidad de la orientación etiológica inicial basada en la valoración de los datos clínicos es motivo de controversia ${ }^{7-9}$.
Por otra parte, la neumonía es una de las primeras causas de infección nosocomial tanto en Chile ${ }^{10}$ como en España $^{11}$. No obstante, la detección de la misma es difícil y requiere un sistema de vigilancia adecuado en todo el hospital. A nivel mundial, la incidencia de la neumonía nosocomial oscila entre 5-10 episodios por cada 1.000 ingresos, dependiendo de las características del hospital ${ }^{12}$, y las tasas más elevadas se producen en las unidades de cuidados intensivos ${ }^{13}$, tasas similares a las descritas en Chile $^{10}$ y en España ${ }^{14}$. El tratamiento antimicrobiano correcto es un factor pronóstico protector, aunque las posibilidades terapéuticas son limitadas. En los últimos años la emergencia de patógenos respiratorios resistentes a muchos antimicrobianos, asociado al uso indiscriminado de estos fármacos, ha potenciado el desarrollo de nuevos antimicrobianos por la industria farmacéutica, efectivos para el tratamiento de estas neumonías ${ }^{15}$.

Tigeciclina es el primer miembro de una nueva clase de antimicrobianos: las glicilciclinas ${ }^{16}$. Presenta un amplio espectro sobre patógenos productores de infecciones complicadas existentes en la comunidad e infecciones nosocomiales, incluyendo bacterias resistentes a $\beta$ lactámicos y a quinolonas ${ }^{17}$. Aunque las indicaciones
Universidad de Sevilla, Sevilla, España.

Facultad de Farmacia Departamento de Farmacología (PMC, VMS)

Hospital Universitario Virgen del Rocío, Sevilla, España. Unidad de Investigación de Enfermedades Infecciosas Pediátricas (JRA) Universidad Pablo de Olavide, Sevilla, España (JMML, SMP).

Los autores declaran no tener conflicto de intereses. Los autores no han recibido ayuda económica de empresa alguna o entidad para la elaboración de este trabajo.

Recibido: 21 de enero de 2013 Aceptado: 9 de octubre de 2013

Correspondencia a: Juan Máximo Molina Linde juanm.molina.ext@ juntadeandalucia.es 
aprobadas por la Food and Drug Administration (FDA) no son exactamente iguales a las de la European Medicines Agency (EMA) ${ }^{18}$, su uso en España se autorizó en 2006 y está limitado a las indicaciones avaladas por los estudios clínicos realizados: infecciones complicadas de piel y tejidos blandos e infecciones intraabdominales. Sin embargo, en la práctica clínica, su utilización se extiende también para el tratamiento de infecciones graves por microorganismos multi-resistentes ${ }^{19,20}$.

En los últimos años se han realizado estudios sobre la utilización de este nuevo antimicrobiano sobre infecciones respiratorias, con resultados diversos y resultados de seguridad controvertidos en algunas ocasiones ${ }^{21,22}$. Aunque hasta la fecha no se hayan descrito efectos graves, tanto la FDA como la EMA recomiendan especial atención ante la reciente aparición de casos de pancreatitis durante su empleo. Actualmente hay algunos ensayos clínicos en marcha que siguen investigando sobre la seguridad y la eficacia de tigeciclina en el tratamiento de este tipo de infecciones.

El objetivo principal de este trabajo ha sido revisar la evidencia que analiza la eficacia y seguridad de tigeciclina en el tratamiento de infecciones asociadas al tracto respiratorio en adultos.

\section{Material y Métodos}

Se ha elaborado una revisión sistemática de la literatura científica para evaluar la eficacia y seguridad de tigeciclina en el tratamiento de infecciones asociadas al tracto respiratorio.

Se desarrolló una búsqueda sistemática en las bases de datos MedLine, Embase, Cochrane Library, CRD (Center for Review and Dissemination) y WOK (Web of Knowledge). También se realizó una búsqueda manual en revistas especializadas de Microbiología, Enfermedades Infecciosas y Neumología. La estrategia de búsqueda se ha elaborado mediante la utilización y combinación de los descriptores MeSH: "tygecicline”, "respiratory infection", "community-acquired pneumonia", "hospitalacquired pneumonia", "safety", "eficacy", "Acinetobacter", "Pseudomonas", y los términos de búsqueda libre "tygacil", "tygecicline", "clinical trial" y "hospital acquired pneumoniae”. La búsqueda se dirigió a la localización de ensayos clínicos con asignación aleatoria. No se realizó restricción de idioma alguna. El período de búsqueda abarcó de enero de 2003 a marzo de 2012.

Criterios de inclusión: Ensayos clínicos con asignación aleatoria, que reclutaran pacientes adultos con infecciones respiratorias, bien con neumonía adquiridas en la comunidad o infecciones respiratorias nosocomiales. La intervención consistía en el tratamiento de la infección respiratoria con tigeciclina frente a otro antimicrobiano como alternativa. Los resultados a determinar fueron de eficacia (eficacia clínica, eficacia microbiológica, test de no inferioridad, resultados por análisis por intención de tratar) y seguridad (efectos adversos locales y sistémicos).

Criterios de exclusión: Estudios en los que la muestra procediera de laboratorio no clínico, ya fuesen colonias, $\mathrm{o}$ bien muestras que no fueran de origen humano, $\mathrm{y}$ estudios en los que no apareciera un grupo comparador o tratamiento placebo.

Para la selección de los estudios, las referencias localizadas fueron inicialmente analizadas por dos investigadores de forma individual, mediante la lectura del título y el resumen, y si cumplían los criterios de inclusión, se localizaba el artículo completo y se valoraba de nuevo su inclusión de manera independiente. El grado de concordancia entre ambos investigadores fue alto.

La evaluación de la calidad metodológica de los ensayos clínicos se realizó mediante el cuestionario elaborado por el Critical Appraisal Skills Programme (CASP) para ensayos clínicos, adaptadas por CASP España (CASPe) ${ }^{23}$.

La comprobación de los resultados descritos en los estudios de valores absolutos y porcentajes, así como la elaboración de tablas y los rangos de resultados se realizó mediante la utilización del programa estadístico SPSS (v18.0). Debido a la heterogeneidad de los estudios, tanto en el tipo de pacientes, intervenciones que se comparaban como en los diseños usados y en los resultados obtenidos, no pudo hacerse un meta-análisis. Se realizó una síntesis cualitativa de los resultados más relevantes, utilizando la valoración crítica de la calidad de los estudios para matizar las conclusiones.

\section{Resultados}

La búsqueda bibliográfica sistemática, encaminada a localizar artículos originales, proporcionó 130 referencias. La primera selección se centró en la eliminación de duplicados (69 exclusiones) y tras la lectura de los títulos y resúmenes se realizaron 52 exclusiones, seleccionándose nueve trabajos que podían cumplir los criterios de inclusión. De los nueve artículos para lectura a texto completo, se excluyeron dos por no ser la intervención adecuada, dos por el tipo de artículo y uno por no ser el objetivo del estudio. Finalmente seleccionamos cuatro estudios correspondientes a ensayos clínicos con asignación aleatoria para realizar la revisión sistemática (Figura 1).

\section{Características descriptivas de los estudios seleccionados}

La Tabla 1 describe las principales características de los cuatro ensayos clínicos incluidos en la revisión sistemática ${ }^{24-27}$. 
Los ensayos clínicos englobaron a un total de 2.155 pacientes con una edad media comprendida entre 50,4 y 57,6 años, sin diferencias significativas entre grupos. Tres estudios $^{24-26}$ incluyeron a pacientes con NAC y un estudio ${ }^{27}$ incluyó a pacientes con neumonía hospitalaria $(\mathrm{NH})$. La intervención fue el tratamiento con tigeciclina IV a dosis inicial de $100 \mathrm{mg}$, seguido de dosis de $50 \mathrm{mg}$ c/12 h.

En los estudios con pacientes NAC ${ }^{24-26}$ el comparador fue el tratamiento con levofloxacina IV a dosis de 500 $\mathrm{mg} / 12-24 \mathrm{~h}$. En el estudio de pacientes con $\mathrm{NH}^{27}$ el tratamiento comparador fue imipenem/cilastatina a dosis de 500-1.000 mg c/8 h. La duración del tratamiento en todos los estudios fue de 7-14 días.

\section{Calidad metodológica de los estudios seleccionados}

La calidad de los ensayos fue alta-moderada. Los resultados de calidad de los estudios se exponen en la Tabla 2. En todos los estudios se describía de manera correcta la pregunta de investigación. Todos los estudios comprendían una asignación aleatoria y doble ciego. Sólo un estudio ${ }^{25}$ describió el método de realización de la aleatorización. Excepto en el estudio de Dartois y cols. ${ }^{24}$, en el resto de trabajos se llevó a cabo un seguimiento completo de los pacientes ${ }^{25-27}$, que se exponen en diagramas de flujo de cada artículo. Los grupos de pacientes en cada grupo eran similares en todos los estudios, aunque uno citó que existían algunas diferencias iniciales entre ambos ${ }^{27}$. Los grupos recibieron en cada estudio un tratamiento igual, aparte de las intervenciones, y en dos trabajos se especificaba que se realizaba ajuste de dosis en pacientes con insuficiencia renal ${ }^{26-27}$.

\section{Principales resultados de los ensayos clínicos incluidos}

Los principales resultados de eficacia y seguridad de los estudios seleccionados para la revisión se detallan en la Tabla 3. En pacientes con NAC la eficacia clínica (EC) de tigeciclina $(88,6-90,6 \%)$ fue no inferior a la de levofloxacina $(85,3-87,2 \%)$. El análisis mediante intención de tratar modificada (ITT-m) también registró una eficacia superior de tigeciclina $(78,0-84,4 \%)$ frente a levofloxacina $(77,8-82,1 \%)$. El "test de no inferioridad" para tigeciclina fue estadísticamente significativo $(\mathrm{p}<0,001)$.

En el estudio de pacientes con $\mathrm{NH}^{27}$, tigeciclina presentó una eficacia de $67,9 \%$ frente a $78,2 \%$ de imipenem/cilastatina. El análisis mediante ITT-m mostró que tigeciclina fue inferior a imipenem/cilastatina $(62,7 \mathrm{vs}$ $67,6 \%$ ). El "test de no inferioridad" de este estudio para tigeciclina fue estadísticamente significativo únicamente para el análisis ITT-m $(\mathrm{p}<0,001)$.

Los principales efectos adversos de tigeciclina descritos fueron gastrointestinales, como náuseas vómitos y diarrea. Otros efectos adversos que se detectaron con menor frecuencia fueron flebitis en el lugar de la inyección, anemia, leucocitosis, aumento de las transaminasas (GOT y GPT) o úlceras en la piel.

Se registraron pocos efectos adversos graves, que no presentaron diferencias entre los grupos. Ninguno de los fallecimientos que se notificaron fue a causa de la utilización de tigeciclina.

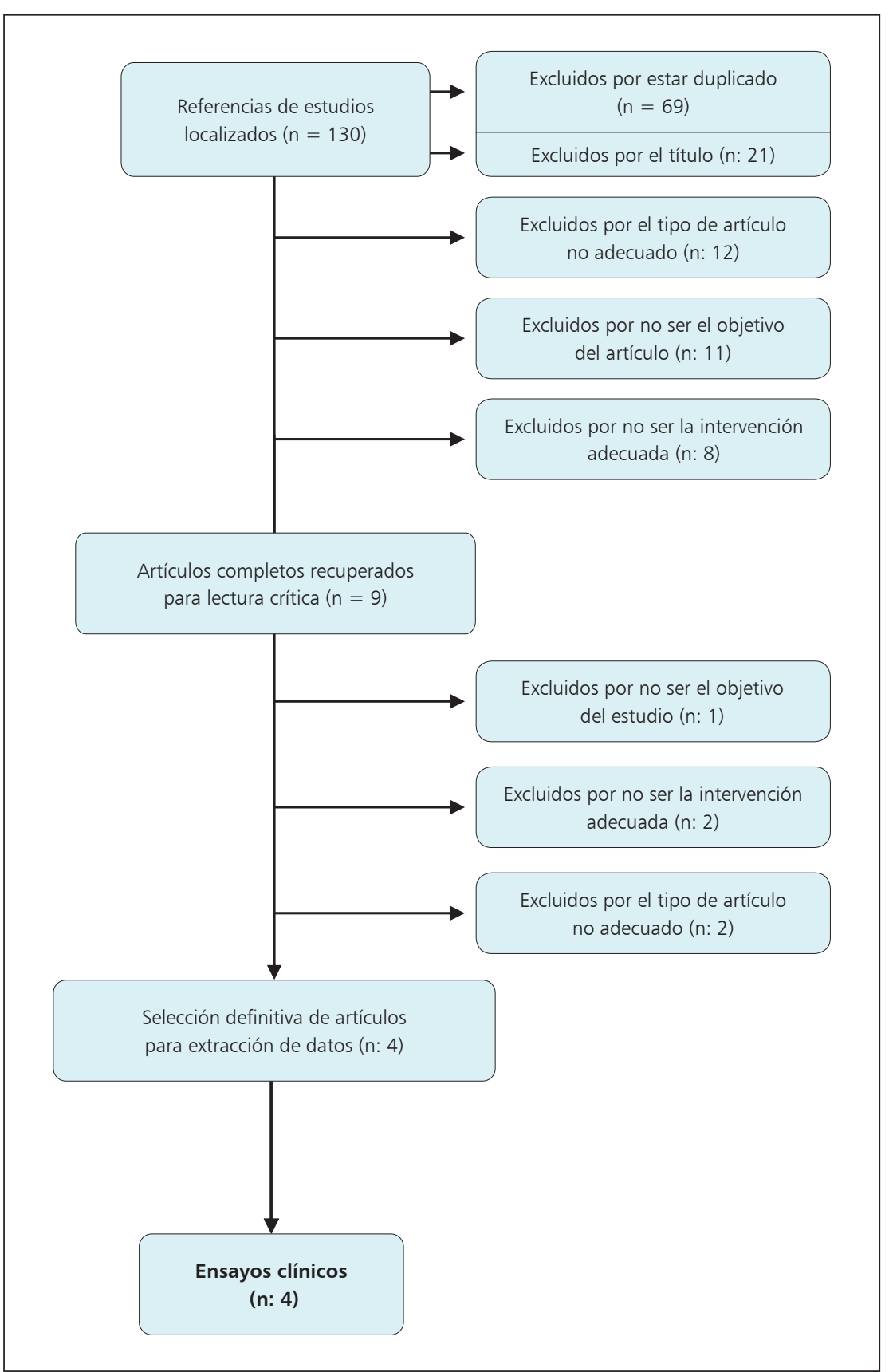

Figura 1. Esquema general de la selección de estudios. Diagrama de flujo: estudios recuperados, valorados, excluidos y motivos de exclusión. 


\begin{tabular}{|c|c|c|c|c|c|c|c|c|}
\hline $\begin{array}{l}\text { Autor } \\
\text { Año }\end{array}$ & $\begin{array}{c}\text { País } \\
\text { Año del estudio } \\
\text { Diseño } \\
\text { Pacientes }\end{array}$ & Otras patologías & $\begin{array}{l}\text { Edad } \\
\text { media }\end{array}$ & $\begin{array}{c}\text { Sexo } \\
\text { (\% hombres) }\end{array}$ & $\begin{array}{c}\text { Duración } \\
\text { del } \\
\text { tratamiento }\end{array}$ & $\begin{array}{l}\text { Intervención } \\
\text { n de pacientes }\end{array}$ & $\begin{array}{c}\text { Comparación } \\
\text { n pacientes }\end{array}$ & Resultados \\
\hline $\begin{array}{l}\text { Bergallo } \\
2009\end{array}$ & $\begin{array}{l}8 \text { p de América } \\
\text { 2003-05 } \\
\text { ECA-dc } \\
418 \text { NAC }\end{array}$ & $\begin{array}{l}\text { 15\% Diabetes mellitus } \\
\text { 15\% EPOC } \\
\text { 7,4\% Patología cardíaca } \\
\text { 10,5\% Alcoholismo }\end{array}$ & $\begin{array}{l}\text { I: } 55,49 \\
\text { C: } 54,10\end{array}$ & $\begin{array}{l}\text { I: } 53,8 \% \\
\text { C: } 62,9 \%\end{array}$ & 7-14 días & $\begin{array}{l}\text { TG } 100 \mathrm{mg}+50 \mathrm{mg} \\
\mathrm{c} / 12 \mathrm{~h} \\
\text { EC: } 138 \\
\text { ec-mITT: } 191 \\
\text { Seguridad: } 208\end{array}$ & $\begin{array}{l}\text { Levofloxacina } 500 \mathrm{mg} \\
\text { c/24 h } \\
\text { EC: } 156 \\
\text { ec-mITT: } 203 \\
\text { Seguridad: } 210\end{array}$ & $\begin{array}{l}\text { EC } \\
\text { ec-mITT } \\
\text { Efectos } \\
\text { adversos }\end{array}$ \\
\hline $\begin{array}{l}\text { Tanaseanu } \\
2009\end{array}$ & $\begin{array}{l}20 \text { p de Europa } \\
\text { África y Asia- } \\
\text { pacífico } \\
2004-05 \\
\text { ECA-dc } \\
434 \text { NAC }\end{array}$ & $\begin{array}{l}\text { 8,5\% Diabetes mellitus } \\
\text { 8,5\% EPOC } \\
\text { 5,7\% Patología cardíaca } \\
\text { 5,7\% ACV } \\
\text { 4,2\% Alcoholismo }\end{array}$ & $\begin{array}{l}\text { I: } 52,8 \\
\text { C: } 50,4\end{array}$ & $\begin{array}{l}\text { I: } 63 \% \\
\text { C: } 64 \%\end{array}$ & 7-14 días & $\begin{array}{l}\text { TG } 100 \mathrm{mg}+50 \mathrm{mg} / 12 \mathrm{~h} \\
\text { EC: } 144 \\
\text { ec-mITT: } 203 \\
\text { Seguridad: } 216\end{array}$ & $\begin{array}{l}\text { Levofloxacina } 500 \mathrm{mg} \\
\text { c/12 ó } 24 \mathrm{~h} \\
\text { EC: } 136 \\
\text { ec-mITT: } 200 \\
\text { Seguridad: } 212\end{array}$ & $\begin{array}{l}\text { EC } \\
\text { ecmlTt } \\
\text { Efectos } \\
\text { adversos }\end{array}$ \\
\hline $\begin{array}{l}\text { Dartois } \\
2008\end{array}$ & $\begin{array}{l}18 \mathrm{p} \text { de Europa } \\
\text { ECA-dc } \\
358 \mathrm{NAC}\end{array}$ & No descrito & & & 7-14 días & $\begin{array}{l}\text { TG } 100 \mathrm{mg}+50 \mathrm{mg} / 12 \mathrm{~h} \\
\text { EC: } 125 \\
\text { ec-mITT: } 177 \\
\text { Seguridad: } 177\end{array}$ & $\begin{array}{l}\text { Levofloxacina } 500 \mathrm{mg} \\
\text { c/12 ó } 24 \mathrm{~h} \\
\text { EC: } 120 \\
\text { ec-mITT: } 181 \\
\text { Seguridad: } 181\end{array}$ & $\begin{array}{l}\text { EC, ecmlTT } \\
\text { TOC }\end{array}$ \\
\hline $\begin{array}{l}\text { Freire } \\
2010\end{array}$ & $\begin{array}{l}31 \text { países } \\
2004-06 \\
\text { ECA-dc } \\
945 \mathrm{NH}\end{array}$ & 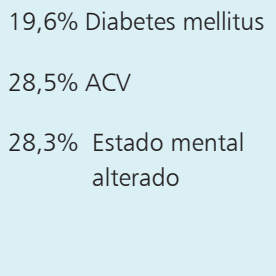 & $\begin{array}{l}\text { I: } 57,2 \\
\text { C: } 57,6\end{array}$ & $\begin{array}{l}\text { I: } 73,1 \% \\
\text { C: } 68,3 \%\end{array}$ & 7-14 días & $\begin{array}{l}\text { TG } 100 \mathrm{mg}+50 \mathrm{mg} / 12 \mathrm{~h} \\
\text { EC: } 268 \\
\text { ec-mITT: } 467 \\
\text { seguridad: } 467\end{array}$ & $\begin{array}{l}\text { Imipenen/cilastatin } \\
\text { 500-1.000 mg c/8 h } \\
\text { CE: } 243 \\
\text { ec-mITT: } 467 \\
\text { seguridad: } 467\end{array}$ & $\begin{array}{l}\text { EC } \\
\text { ecmlTt } \\
\text { Efectos } \\
\text { adversos }\end{array}$ \\
\hline
\end{tabular}

Tabla 2. Calidad de los ensayos clínicos seleccionados según la escala CASPe

\begin{tabular}{|c|c|c|c|c|c|c|}
\hline \multirow[b]{2}{*}{ Artículo } & \multicolumn{6}{|c|}{ Validez de la intervención } \\
\hline & $\begin{array}{l}\text { Definición clara } \\
\text { de la pregunta }\end{array}$ & $\begin{array}{l}\text { Asignación aleatoria } \\
\text { de pacientes }\end{array}$ & $\begin{array}{l}\text { Seguimiento completo } \\
\text { de los pacientes }\end{array}$ & Enmascaramiento & $\begin{array}{l}\text { Grupos similares } \\
\text { al comienzo }\end{array}$ & $\begin{array}{l}\text { Tratamiento igual } \\
\text { de los grupos }\end{array}$ \\
\hline $\begin{array}{l}\text { Bergallo } \\
2009\end{array}$ & Sí & Sí, no método & Sí & Sí, doble & Sí & Sí, ajuste en IR \\
\hline $\begin{array}{l}\text { Tanaseanu } \\
2009\end{array}$ & Sí & Sí & Sí & Sí, doble & Sí & Sí \\
\hline $\begin{array}{l}\text { Dartois } \\
2008\end{array}$ & Sí & Sí, no método & ND & Sí, doble & Sí & Sí \\
\hline $\begin{array}{l}\text { Freire } \\
2010\end{array}$ & Sí & Sí, no método & Sí & Sí, doble & Sí & Sí, ajuste en IR \\
\hline
\end{tabular}




\begin{tabular}{|c|c|c|c|c|}
\hline \multirow{3}{*}{$\begin{array}{l}\text { Autor } \\
\text { Año } \\
\text { Bergallo } \\
2009\end{array}$} & \multirow{2}{*}{$\begin{array}{l}\text { Efectividad clínica } \\
\begin{array}{ll}\text { I: } 90,6 \% & \text { p: } 0,4570\end{array}\end{array}$} & \multirow{2}{*}{$\begin{array}{l}\text { Efectividad clínica con intención de } \\
\text { trata modificada (m-ITT) } \\
\begin{array}{ll}\text { l: } 78,0 & \text { p: } 1,0\end{array}\end{array}$} & \multicolumn{2}{|l|}{ Efectos adversos ( $1 \%$ vs C\%) } \\
\hline & & & Alguno: 51,4 vs 45,7 & p: NS \\
\hline & C: $87,2 \%$ & C: 77,8 & Náuseas : 16,3 vs 5,7 & $p<0,05$ \\
\hline & Eficacia de tigeciclina: & Eficacia de tigeciclina: & Vómitos: 12,0 vs 1,9 & $p<0,05$ \\
\hline & Test de no inferioridad $\mathrm{p}:<0,001$ & Test de no inferioridad $\mathrm{p}:<0,001$ & Diarrea: 8,2 vs 4,3 & p: NS \\
\hline & & & Aumento de GOT, GPT: 3,6 vs 10,0 & $p<0,05$ \\
\hline & & & Flebitis: 7,2 vs 7,6 & p: NS \\
\hline & & & \multicolumn{2}{|l|}{ EAG: no descritos } \\
\hline & & & \multicolumn{2}{|c|}{ fallecimientos: 2,4 vs 2,8 (ninguno por los tratamientos) } \\
\hline Tanaseanu & I: $88,9 \%$ & I: $83,7 \%$ & Alguno: 62,5 vs 47,2 & p: 0,002 \\
\hline \multirow[t]{8}{*}{2009} & C: $85,3 \%$ & C: $81,5 \%$ & Náuseas: 26,9 vs 8,5 & $\mathrm{p}:<0,001$ \\
\hline & Eficacia de tigeciclina: & Eficacia de tigeciclina: & Vómito: 16,7 vs 6,6 & p: 0,001 \\
\hline & Test de no inferioridad $\mathrm{p}:<0,001$ & Test de no inferioridad $\mathrm{p}:<0,001$ & Diarrea: 7,4 vs 8,0 & p: 0,85 \\
\hline & & & Aumento de GOT, GPT: 1,9 vs 4,2 & p: 0,169 \\
\hline & & & Leucocitosis: 6,9 vs 0,9 & p: 0,002 \\
\hline & & & Hipocalemia: 0,5 vs 3,8 & p: 0,019 \\
\hline & & & \multicolumn{2}{|l|}{ EAG: 8,3 vs 9,9} \\
\hline & & & \multicolumn{2}{|c|}{ fallecimientos: 3,2 vs 2,3 (ninguno por los tratamientos) } \\
\hline Dartois & I: $88,6 \%$ & I: $84,4 \%$ & \multicolumn{2}{|l|}{ Efectos adversos: no descritos } \\
\hline \multirow[t]{3}{*}{2008} & C: $85,8 \%$ & C: $82,1 \%$ & \multirow{3}{*}{\multicolumn{2}{|c|}{$\begin{array}{l}\text { EAG: } 1,6 \text { vs } 1,1 \\
\text { fallecimientos: no descritos }\end{array}$}} \\
\hline & Eficacia de tigeciclina: & Eficacia de tigeciclina: & & \\
\hline & Test de no inferioridad $\mathrm{p}:<0,001$ & Test de no inferioridad $\mathrm{p}:<0,001$ & & \\
\hline Freire & I: $67,9 \%$ & I: $62,7 \%$ & Alguno: 78,8 vs 78,6 & p: 1,0 \\
\hline \multirow[t]{8}{*}{2010} & C: $78,2 \%$ & C: $67,6 \%$ & Náuseas: 15,4 vs 7,9 & $\mathrm{p}:<0,001$ \\
\hline & Eficacia de tigeciclina: & Eficacia de tigeciclina: & Vómitos: 12,6 vs 7,3 & p: 0,008 \\
\hline & Test de no inferioridad $\mathrm{p}: 0,120$ & Test de no inferioridad $p:<0,001$ & Diarrea: 10,5 vs 15,5 & p: 0,049 \\
\hline & & & Anemia: 6,9 vs 9,6 & p: 0,153 \\
\hline & & & Úlcera de piel: 5,4 vs 2,1 & p: 0,015 \\
\hline & & & EAG: 26,1 vs 25,7 & p: 0,940 \\
\hline & & & EAG respiratorio: 7,1 vs 11,6 & $p<0,05$ \\
\hline & & & \multicolumn{2}{|c|}{ fallecimientos 14,1 vs 12,2 (sólo 3 por los tratados) } \\
\hline
\end{tabular}

\section{Discusión}

Las infecciones respiratorias por microorganismos multi-resistentes han emergido en las últimas décadas, sobre todo en el medio hospitalario y especialmente con bacterias gramnegativas con mecanismos de resistencia. Asimismo, el desarrollo de nuevos antimicrobianos, como tigeciclina, con amplio espectro de actividad sobre diversos microorganismos todavía no se considera óptimo, debido a las posibles resistencias.

Esta revisión sintetiza la información actual existente sobre el tratamiento de infecciones respiratorias hospita- larias y NAC mediante la utilización de tigeciclina como antimicrobiano.

Los resultados de este trabajo reflejan que la eficacia de tigeciclina resulta adecuada para el tratamiento de infecciones respiratorias, según los estudios analizados, ya que el "test de no inferioridad" frente a otro antimicrobiano comparado, obtuvo significación estadística. Con los datos que hemos analizados, los mejores resultados se reflejan en estudios con pacientes con $\mathrm{NAC}^{24-26}$, ya que en todos los casos la eficacia de tigeciclina fue no inferior al fármaco con el que se comparaba, mediante el "test de no inferioridad". Al analizar el estudio de pacientes con $\mathrm{NH}$ 
la tigeciclina mostró una eficacia ligeramente inferior al antimicrobiano comparador ${ }^{27}$.

Tigeciclina ha presentado un buen perfil de seguridad, si bien se han encontrado algunos eventos adversos gastrointestinales; sin embargo, es bien tolerada y son escasos los abandonos o fracasos terapéuticos en pacientes con patologías respiratorias ${ }^{28}$.

Esta revisión sistemática ha presentado una serie de limitaciones. En primer lugar se ha visto influenciada por el escaso número de estudios y la heterogeneidad de éstos, que abordan la pregunta de investigación planteada al principio del trabajo de diferente manera. Así, se ha localizado un solo estudio que aborda la $\mathrm{NH}$, por lo que los resultados no han podido ser comparados con otros. No se ha considerado la inclusión de otro tipo de estudios no controlados para tratar de sintetizar, con la máxima homogeneidad y calidad metodológica posible, los resultados acontecidos. Otra limitación de este trabajo es el potencial sesgo de publicación, ya que se excluyeron trabajos aún no publicados, estudios en fase clínica en desarrollo, literatura gris e informes de casas comerciales. Este sesgo se ha tratado de evitar al extender la búsqueda en varias bases de datos y realizarla sin restricción de idiomas. Con el fin de evitar un posible sesgo en la aplicación de los criterios de selección, éstos se definieron a priori. Otra posible limitación a la hora de valorar los resultados es que los ensayos han sido financiados por la industria farmacéutica, por lo que se deben interpretar las conclusiones con cautela.

Los resultados obtenidos de los artículos analizados están limitados por una serie de factores y presentan algunos problemas metodológicos, tanto de validez interna como externa, que pueden ser la causa de las diferencias acusadas entre las dosis y que hacen que presenten una calidad alta-moderada. Los factores más importantes han sido la falta explicación de asignación en forma aleatoria, características iniciales de los grupos de pacientes y el tratamiento desigual de los mismos según otras patologías asociadas y el tratamiento desigual. La determinación de resultados también ha presentado heterogeneidad según las determinaciones ensayadas, ya que no en todos se midieron las mismas variables de resultado. También la intervención se realizaba de manera diferente (posología, dosis, tipos de pacientes), así como la posterior selección e interpretación de datos.

Nuestros resultados coinciden parcialmente con los resultados de otros trabajos de revisión que evaluaban tigeciclina en infecciones ${ }^{21,22,29-31}$. Pero en ninguno de estos trabajos se evaluaba de manera específica la eficacia y seguridad de tigeciclina exclusivamente en infecciones respiratorias.

La introducción de tigeciclina, la primera glicilciclina, podría proporcionarnos una opción adicional para el manejo de estas complejas infecciones. Todavía la EMA no ha autorizado su utilización en este tipo de infecciones, por lo que, aunque los resultados así lo avalen, su administración terapéutica está actualmente restringida a patologías concretas. Sin embargo, probablemente su nicho real de utilización estaría orientado hacia el manejo de infecciones del ámbito intrahospitalario, sobre todo en el contexto de cuidados críticos ${ }^{28}$. Según la información revisada, tigeciclina se presenta con una utilidad potencial en el manejo de NHs, ya que ha demostrado ser "no inferior" a otros antimicrobianos en los estudios evaluados. Sin embargo, se debe observar cierta precaución y evitar en la práctica clínica diaria su uso inapropiado o sobreuso, pues indudablemente resultará en una mayor resistencia bacteriana, ya comunicada en las experiencias clínicas de algunos estudios, donde se verifica la aparición de cepas con susceptibilidad reducida o no susceptibles a tigeciclina, a veces durante el curso del tratamiento con el mismo antimicrobiano. Sería recomendable la realización de nuevos ensayos clínicos, con asignación aleatoria y controlados, realizados por investigadores independientes y en contextos clínicos más amplios, que incluyeran adultos mayores, con co-morbilidad múltiple y diferentes etiologías microbianas y estimaciones de la gravedad basados en el índice de gravedad de la neumonía.

También sería importante para los especialistas en enfermedades infecciosas y profesionales farmacéuticos cautelar el uso prudente de este nuevo antibacteriano, una vez se apruebe su indicación para este tipo de infecciones, reservándolo fundamentalmente como una terapia de rescate para patógenos que presenten patrones de multiresistencia.

\section{Resumen}

Introducción: Tigeciclina está indicada en el tratamiento de infecciones complicadas de piel, tejidos blandos e intra-abdominales. Su utilización podría extenderse para neumonías adquiridas en la comunidad (NAC) y neumonías hospitalarias (NH). El objetivo ha sido evaluar la eficacia y seguridad de tigeciclina en el tratamiento de infecciones respiratorias. Material y Métodos: Revisión sistemática (2012). Se realizaron búsquedas en MedLine, Embase, Cochrane Library, CRD y WOK. Se localizaron ensayos clínicos de adultos con infección respiratoria, tratados mediante tigeciclina. La calidad de los estudios se valoró mediante los criterios CASPe. Resultados: Se seleccionaron cuatro ensayos clínicos de calidad altamoderada. Tres estudios incluyeron pacientes con NAC y un estudio a pacientes con NH. En pacientes con NAC la eficacia de tigeciclina $(88,6-90,6 \%)$ fue no inferior a la de levofloxacina $(85,3-87,2 \%)$. El "test de no inferioridad" fue estadísticamente significativo $(p<0,001)$. En el estudio de pacientes con $\mathrm{NH}$, tigeciclina presentó una 
eficacia de $67,9 \%$ frente a $78,2 \%$ de imipenem/cilastatina. Los principales efectos adversos fueron gastrointestinales. Conclusiones: la eficacia de tigeciclina es no inferior a la de levofloxacina en pacientes con NAC, pero inferior a imipenem en pacientes con NH. Tigeciclina ha demostrado no inferioridad frente a los otros antimicrobianos testados. Tigeciclina demuestra tener un buen perfil de seguridad.

\section{Referencias bibliográficas}

1.- Falguera M, Gudiol F, Sabriá M, ÁlvarezLerma F, Cordero E. Infecciones en el tracto respiratorio inferior. Protocolos clínicos SEIMC. [Internet] 2000 [citado 14 diciembre 2012]. Disponible en: http://www.seimc.org/ documentos/protocolos/clinicos/

2.- Ministerio de Salud de Chile. Programa de Infecciones Respiratorias Agudas (IRA). Departamento de Estadísticas e Información de Salud, Ministerio de Salud de Chile. [Internet] [citado 26 agosto de 2013]. Disponible en: http://www.deis.cl.

3.- Sociedad Chilena de Enfermedades Respiratorias y Sociedad Chilena de Infectología. Manejo de la neumonía del adulto adquirida en la comunidad. Resumen del consenso nacional. Rev Med Chile 2005; 133 : 953-67.

4.- Monge V, San Martín V M, González A. The burden of community-acquired pneumonia in Spain. Eur J Public Health 2001; 4: 362-4.

5.- Vila M, Bello S. Vacuna antineumocócica: indicaciones, momento y resultados. Arch Bronconeumol 2004; 40 (Supl 3): 43-50.

6.- Cohen R. The need for prudent use of antibiotics and routine use of vaccines. Clin Microbiol Infect 2009; 15 (Suppl 3): 21-3.

7.- Martín A A, Moreno-Pérez D, Miguélez S A, Gianzo J A, García M L, Murua J K, et al Etiología y diagnóstico de la neumonía adquirida en la comunidad y sus formas complicadas. An Pediatr (Barc) 2012; 76: 162. e1-18.

8.- Don M, Canciani M, Korppi M. Communityacquired pneumonia in children: what's old? What's new? Acta Paediatrica 2010; 99: 1602-8

9.- Woodhead M, Blasi F, Ewig S, Garau J, Huchon G, Ieven M, et al. Guidelines for the management of adult lower respiratory tract infections-full version. Clin Microbiol Infect 2011; 17 (Suppl 6): E1-59.

10.- Gobierno de Chile. Ministerio de Salud. Hospital Del Salvador. Oficina de Calidad y Seguridad del Paciente. Norma Prevención Neumonía Nosocomial Asociada a VMI. [Internet] [citado el 26 agosto de 2013]. Disponible en: http:// mx.hsalvador.cl/home/archivos@hsalvador. $\mathrm{cl} /$ www/oficina\%20calidad\%20y\%20seguridad\%20de \%20pacientes/PREVNEUMONIANOSOCOMIAL.pdf

11.- Sopena N. Predicción clínica de la neumonía nosocomial por Staphylococcus aureus resistente a la meticilina. Med Clin (Barc) 2012; 138: 112-3.

12.- Craven DE, Chroneou A. Nosocomial pneumonia. En: Mandell G L, Bennett J E, Dolin R, editors. Mandell, Douglas \& Bennett's Principles and Practice of Infectious Diseases. 7th ed., Philadelphia: Elsevier Churchill Livingstone; 2009. p. 3717-24.

13.- American Thoracic Society; Infectious Diseases Society of America. Guidelines for the management of adults with hospital-acquired, ventilator-associated, and healthcare-associated pneumonia. Am J Respir Crit Care Med 2005; 171: 388-416.

14.- Sociedad Española de Medicina Preventiva, Salud Pública e Higiene. Estudio de prevalencia de las infecciones nosocomiales en los hospitales españoles. EPINE Informe 2011. [Internet] abril de 2011 [citado 20 diciembre de 2012]. Disponible en: http://hws.vhebron. net/epine/Descargas/EPINE\%202011\%20 ESPAÑA\%20Resumen.pdf

15.- Karageorgopoulos D E, Falagas M E. New antibiotics: optimal use in current clinical practice. Int J Antimicrob Agents 2009; 34 (Suppl 4): S55-62.

16.- Wyeth-Pfizer. Tigecycline (Tygacil ${ }^{\circledR}$ ) product information. Wyeth Pharmaceuticals Inc., Philadelphia, PA, June 2005.

17.- Bertrand X, Dowzicky M J. Antimicrobial susceptibility among gram-negative isolates collected from intensive care units in North America, Europe, the Asia-Pacific Rim, Latin America, the Middle East, and Africa between 2004 and 2009 as part of the Tigecycline Evaluation and Surveillance Trial. Clin Ther 2012; 34: 124-37.

18.- Reinert R R, Low D E, Rossi F, Zhang X, Wattal C, Dowzicky M J. Antimicrobial susceptibility among organisms from the Asia/ Pacific Rim. Europe and Latin and North America collected as part of TEST and the in vitro activity of tigecycline. J Antimicrob Chemother 2007; 60: 1018-29.

19.- Betriu C, Rodríguez-Avial I, López F, Picazo J J. Comparative study of the in vitro activity of tigecycline against multiresistant Enterococcus faecium isolates. Enferm Infecc Microbiol Clin 2009; 27: 302-3.

20.- Sorlozano A, Gutiérrez J, Román E, de Dios L J, Roman J, Liebana J, et-al. A comparison of the activity of tigecycline against multiresistant clinical isolates of Staphylococcus aureus and Streptococcus agalactiae. Diagn
Microbiol Infect Dis 2007; 58: 487-9.

21.- Cai Y, Wang R, Liang B, Bai N, Liu Y. Systematic review and meta-analysis of the effectiveness and safety of tigecycline for treatment of infectious disease. Antimicrob Agents Chemother 2011; 55: 1162-72.

22.- Tasina E, Haidich AB, Kokkali S, Arvanitidou M. Efficacy and safety of tigecycline for the treatment of infectious diseases: a meta-analysis. Lancet Infect Dis 2011; 11: 834-44.

23.- Guías CASPe de Lectura Crítica de la Literatura. Alicante: CASPe España, Coordinación General. [Internet] 2005 [citado 10 diciembre de 2012]. Disponible en: http:// www.redcaspe.org/homecasp.asp.

24.- Dartois N, Castaing N, Gandjini H, Cooper A. Tigecycline 313 Study Group. Tigecycline versus levofloxacin for the treatment of community acquired pneumonia: European experience. J Chemother 2008; 20 (Suppl 1): 28-35.

25.- Tanaseanu C, Milutinovic S, Calistru P I, Strausz J, Zolubas M, Chernyak V, et al. Efficacy and safety of tigecycline versus levofloxacin for community-acquired pneumonia. BMC Pulmonary Medicine 2009; 9: 44-56.

26.- Bergallo C, Jasovich A, Teglia O, Oliva M E, Lentnek A, Wouters L, et al. Safety and efficacy of intravenous tigecycline in treatment of community-acquired pneumonia: results from a double-blind randomized phase 3 comparison study with levofloxacin. Diagn Microbiol Infect Dis 2009; 63: 52-61.

27.- Freire A T, Melnyk V, Kim M J, Datsenko O, Dzyublik O, Glumcher F, et al. Comparison of tigecycline with imipenem/cilastatin for the treatment of hospital-acquired pneumonia. Diagn Microbiol Infect Dis 2010; 68: 140-51.

28.- Alexis Diomedi P. Tigeciclina. Una revisión sistemática de la experiencia clínica en sus primeros años de uso. Rev Chilena Infectol 2009; 26: 114-25.

29.- Zhanel G G, Homenuik K, Nichol K, Noreddin A, Vercaigne L, Embil J, et al. The glycylcyclines: a comparative review with the tetracyclines. Drugs 2004; 64: 63-88.

30.- Rello J. Pharmacokinetics, pharmacodynamics, safety and tolerability of tigecycline. J Chemother 2005; 17 (Suppl 1): 12-22.

31.- Nathwani D. Tigecycline: clinical evidence and formulary positioning. Int J Antimicrob Agents 2005; 25: 185-92. 